\title{
Correction to: A large and persistent outbreak of typhoid fever caused by consuming contaminated water and street- vended beverages: Kampala, Uganda, January - June 2015
}

Steven Ndugwa Kabwama1*, Lilian Bulage', Fred Nsubuga', Gerald Pande', David Were Oguttu', Richardson Mafigiri', Christine Kihembo ${ }^{1}$, Benon Kwesiga', Ben Masiira', Allen Eva Okullo', Henry Kajumbula², Joseph KB Matovu ${ }^{3}$, Issa Makumbi ${ }^{4}$, Milton Wetaka ${ }^{4}$, Sam Kasozi ${ }^{4}$, Simon Kyazze ${ }^{4}$, Melissa Dahlke ${ }^{4}$, Peter Hughes ${ }^{5}$, Juliet Nsimire Sendagala ${ }^{5}$, Monica Musenero ${ }^{6}$, Immaculate Nabukenya ${ }^{6}$, Vincent R. Hill ${ }^{7}$, Eric Mintz ${ }^{7}$, Janell Routh ${ }^{7}$, Gerardo Gómez ${ }^{7}$, Amelia Bicknese ${ }^{7}$ and Bao-Ping Zhu ${ }^{7,8}$

After publication of the article [1], it has been brought to our attention that the author Joseph Matovu is missing his initials from his full professional title 'Joseph KB Matovu'.

\begin{abstract}
Author details
'Uganda Public Health Fellowship Program, Field Epidemiology Track, Ministry of Health, Kampala, Uganda. ${ }^{2}$ Makerere University College of Health Science Microbiology Laboratory, Kampala, Uganda. ${ }^{3}$ Makerere University School of Public Health, Kampala, Uganda. ${ }^{4}$ Public Health Emergency Operations Center, Ministry of Health, Kampala, Uganda. ${ }^{5}$ Medical Research Council, Kampala, Uganda. ${ }^{6}$ Epidemiology and Surveillance Division, Ministry of Health, Kampala, Uganda. 'US Centers for Disease Control and Prevention, Atlanta, GA, USA. ${ }^{8}$ US Centers for Disease Control and Prevention, Kampala, Uganda.
\end{abstract}

Received: 28 September 2017 Accepted: 2 October 2017

Published online: 18 October 2017

\section{Reference}

1. Kabwama S, Bulage L, Nsubuga F, Pande G, Oguttu D, Mafigiri R, et al. A large and persistent outbreak of typhoid fever caused by consuming contaminated water and street-vended beverages: Kampala, Uganda, January - June 2015. BMC Public Health. 2017;17:1. doi:10.1186/s12889-016-4002-0.

\footnotetext{
* Correspondence: skabwama@musph.ac.ug

${ }^{1}$ Uganda Public Health Fellowship Program, Field Epidemiology Track,

Ministry of Health, Kampala, Uganda

Full list of author information is available at the end of the article
} 